\title{
Photonic Time Stretch and Its Application to Analog-to-Digital Conversion
}

\author{
F. Coppinger, A. S. Bhushan, and B. Jalali, Senior Member, IEEE
}

\begin{abstract}
We demonstrate a new concept for analog-to-digital (A/D) conversion based on photonic time stretch. The analog electrical signal is intensity modulated on a chirp optical waveform generated by dispersing an ultrashort pulse. The modulated chirped waveform is dispersed in an optical fiber, leading to the stretching of its envelope. We have derived analytical expressions for the stretch factor and the resolution of the system. An analogto-digital converter (ADC) consisting of the photonic time-stretch preprocessor and a 1-Gsample/s electronic ADC is demonstrated. This technique is promising for $A / D$ conversion of ultrafast signals and, hence, for realization of the digital receiver.
\end{abstract}

Index Terms - Analog-to-digital conversion, chirp pulses, mode-locked laser, time stretching.

\section{INTRODUCTION}

I T IS WIDELY recognized that the analog-to-digital converter (ADC) represents the key bottleneck in modern high-performance communication and radar systems. Rapid progress in CMOS technology combined with advances in parallel computing architectures has made the 100-Gflops digital processor a reality. However, the wealth of new system capabilities offered by such processors cannot be fully exploited due to the limited performance of ADC's. While electronic ADC's continue to improve, the rate of improvement is too slow compared to that of digital signal processors (DSP's). This trend underscores the need for new concepts leading to revolutionary improvements in ADC performance.

Such improvements may be obtained if one can reduce the speed of the analog signal prior to digitization [1], [2]. If the signal can be stretched in time without distortion, then the effective sampling rate and the input bandwidth of the ADC is increased in proportion to the stretch factor. In the case of a continuous-time operation, the input signal will have to be segmented and interleaved into parallel channels before each segment is stretched in time.

Recently, we have demonstrated that electrical signals can be stretched in time using chirped optical pulses and dispersion in optical fibers [3]. The system is far simpler to implement, and has demonstrated superior performance to that of the traditional time manipulation technique [4], [5]. This paper presents, for the first time, theoretical analysis for the stretch factor and the resolution of such a system. The expression

Manuscript received October 12, 1998; revised March 12, 1999. This work was supported by the DARPA PACT Program under a grant monitored by SPAWAR.

The authors are with the Optoelectronic Circuits and Systems Laboratory, Department of Electrical Engineering, University of California, Los Angeles, CA 90095-1594 USA.

Publisher Item Identifier S 0018-9480(99)05200-X.

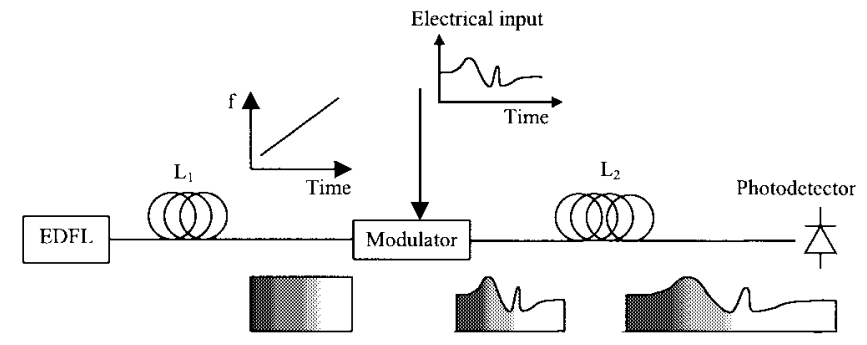

Fig. 1. Schematic description of the time-stretch system.

for the stretch factor is verified by experimental results. The paper also describes preliminary ADC experiments consisting of the photonic time-stretch preprocessor and a 1-Gsample/s electronic ADC. Section V discusses derivatives of this system leading to the concept of wavelength division sampling (WDS), and the time-stretched WDS (TSWDS).

\section{System DESCRIPTION AND EXPERIMENTAL RESUltS}

Fig. 1 describes schematically the technique used for time stretching of electrical signals. A compressed pulse originated in an erbium-doped fiber laser (EDFL) is dispersed in a spool of fiber of length $L_{1}$, generating a chirped optical waveform. The electrical signal is then intensity modulated onto the chirp waveform in a Mach-Zehnder modulator leading to direct time-to-wavelength correspondence. The modulated waveform is then further dispersed in a second spool of fiber of length $L_{2}$, stretching the envelope in time.

The experiments used 160 fs pulses along with a fiber length of $L_{1}=1.1 \mathrm{~km}$ to generate the chirped waveform. This waveform was modulated in a $12-\mathrm{GHz} \mathrm{LiNbO}_{3}$ Mach-Zehnder intensity modulator. A random signal was applied to the modulator in order to generate a non-Gaussian test signal. The modulated chirped waveform was dispersed in a second spool of fiber of length $L_{2}$, stretching the electrical signal. Fig. 2(b)-(d) represents the signal, measured on a sampling oscilloscope, after propagation through $L_{2}=2.2$, 5.5 , and $7.6 \mathrm{~km}$, respectively. As can be seen, the envelope of the signal is stretched as it propagates through the second dispersive element. The measured stretch factors are 3, 6, and 8, respectively. For comparison, Fig. 2(a) shows the test waveform before the second fiber $\left(L_{2}\right)$.

In an ideal time-magnification system [4], [5], the signal is subjected to a sequence of dispersion followed by quadratic phase modulation (or linear frequency ramp) and a second dispersion sequence. The concept of time lens has been widely studied theoretically, and the expression of the focal time and 
(a)

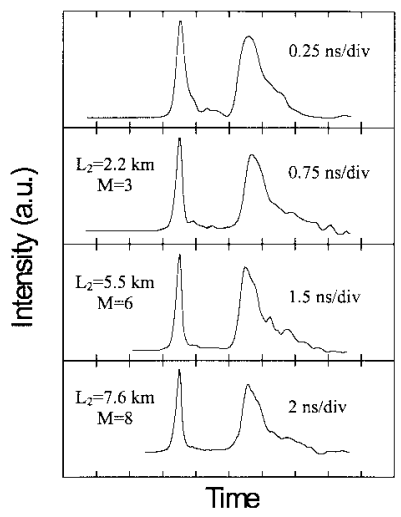

Fig. 2. Envelope of the chirp optical pulse (a) after the modulator and after propagation through (b) $2.2 \mathrm{~km}$, (c) $5.5 \mathrm{~km}$, and (d) $7.6 \mathrm{~km}$ of fiber, showing stretch factors of 3,6 , and 8 , respectively.

requirements for temporal imaging leading to a focussed image have been obtained [6]. The experimental demonstrations, however, had limited success due to the difficulty of obtaining high-rate quadratic phase modulation or highly dispersive elements [7], [8]. Furthermore, the experiments were limited to manipulation of simple optical pulses or digital electrical signals [7], [8]. In the new time-stretching technique described here, the signal is not dispersed prior to mixing with the frequency ramp (see Fig. 1), leading to a nonfocused image in time. A nonfocused image manifests itself as classical dispersion, i.e., slow phenomena (low frequency) will appear clearly, while fast phenomena will be filtered out. This nonfocused image will limit the maximum modulation frequency that can be handled by the time-stretch processor. As will be seen in the following mathematical analysis, the maximum frequency resolvable depends on the chirp rate and exceeds $50 \mathrm{GHz}$ for our experimental system.

\section{ANALYSIS}

To understand and analyze the system, we assume an ultrashort transform-limited Gaussian pulse. After propagation through the first fiber of length $L_{1}$, the electric field can be represented by [9]

$$
E_{\mathrm{ch}}\left(L_{1}, t\right)=\exp \left(\frac{-t^{2}}{\tau^{2}}\right) \times \exp \left(-\frac{j t^{2}}{2 L_{1} \beta_{2}}\right)
$$

where $\beta_{2}$ is the second derivative of the propagation constant $\beta$ with respect to the angular frequency, and $2 \tau$ is the $1 / e$ width of the Gaussian pulse after dispersion. Here, we assume $L_{1} \beta_{2} / \tau^{2} \ll 1$ [9]. Physically, this approximation means that the pulse duration before the first dispersion element is much smaller than the pulse duration after the first dispersion. This is always the case for subpicosecond pulses propagating in a few hundred meters of fiber. In the frequency domain, the electric field is represented by

$E_{\mathbf{c h}}\left(L_{1}, f\right)=\frac{\sqrt{\pi}}{\left|\sqrt{\tau^{-2}+j / 2 L_{1} \beta_{2}}\right|} \times \exp \left(\frac{-\pi^{2} f^{2}}{\tau^{-2}+j / 2 L_{1} \beta_{2}}\right)$.

This chirp pulse is intensity modulated in the $\mathrm{LiNbO}_{3}$ modulator with a sinusoid at frequency $f_{m}$. The modulation depth $a$ is small, such that harmonics of $f_{m}$ can be neglected. The modulated electric field is expressed by

$$
E_{\mathbf{i n}}\left(L_{1}, t\right)=E_{\mathbf{c h}}\left(L_{1}, t\right) \times\left[1+a \cos \left(2 \pi f_{m} t\right)\right] .
$$

The modulated electric field can be represented in the frequency domain as follows:

$$
\begin{aligned}
E_{\text {in }}\left(L_{1}, f\right)= & E_{\mathbf{c h}}\left(L_{1}, f\right) \\
& *\left[\delta(f)+\frac{a}{2} \cdot\left\{\delta\left(f-f_{m}\right)+\delta\left(f+f_{m}\right)\right\}\right]
\end{aligned}
$$

where " $*$ " denotes the convolution operation. Thus, the output electric field after dispersion through $\left(L_{1}+L_{2}\right)$ meters of fiber is given by

$$
E_{\text {out }}\left(L_{1}+L_{2}, f\right)=E_{\text {in }}\left(L_{1}, f\right) \times \exp \left(j 2 \pi^{2} L_{2} \beta_{2} f^{2}\right)
$$

or

$$
\begin{aligned}
& E_{\text {out }}\left(L_{1}+L_{2}, f\right) \\
&= \frac{\sqrt{\pi}}{\left|\sqrt{\tau^{-2}+j / 2 L_{1} \beta_{2}}\right|} \\
& \times\left[\exp \left(\frac{-\pi^{2} f^{2}}{\tau^{-2}+j / 2 L_{1} \beta_{2}}\right)+\frac{a}{2}\right. \\
& \cdot\left\{\exp \left(\frac{-\pi^{2}\left(f-f_{m}\right)^{2}}{\tau^{-2}+j / 2 L_{1} \beta_{2}}\right)\right. \\
&\left.\left.+\exp \left(\frac{-\pi^{2}\left(f+f_{m}\right)^{2}}{\tau^{-2}+j / 2 L_{1} \beta_{2}}\right)\right\}\right] \\
& \times \exp \left(j 2 \pi^{2} L_{2} \beta_{2} f^{2}\right) .
\end{aligned}
$$

The electric field in time domain is given by (see the Appendix)

$$
\begin{aligned}
E_{\text {out }}\left(L_{1}+L_{2}, t\right) & \sqrt{\frac{L_{1}}{L_{1}+L_{2}} \times \exp \left[\frac{-t^{2}}{\left\{\tau\left(L_{1}+L_{2}\right) / L_{1}\right\}^{2}}\right]} \\
\times & \exp \left[\frac{-j t^{2}}{2\left(L_{1}+L_{2}\right) \beta_{2}}\right] \\
\times & {\left[\begin{array}{l}
1+\frac{a}{2}\left\{\exp \left(j 2 \pi f_{m} t \cdot \frac{L_{1}}{L_{1}+L_{2}}\right)+\right. \\
\exp \left(-j 2 \pi f_{m} t \cdot \frac{L_{1}}{L_{1}+L_{2}}\right)
\end{array}\right\} } \\
& \left.\times \exp \left(j 2 \pi^{2} L_{2} \beta_{2} f_{m}^{2} \cdot \frac{L_{1}}{L_{1}+L_{2}}\right)\right] .
\end{aligned}
$$

The light intensity detected by the photodetector is, therefore,

$$
\begin{array}{r}
I_{\text {out }}\left(L_{1}+L_{2}, t\right) \\
\propto E_{\text {out }}\left(L_{1}+L_{2}, t\right) \times E_{\text {out }}^{*}\left(L_{1}+L_{2}, t\right) \\
=\frac{L_{1}}{L_{1}+L_{2}} \times \exp \left(-\frac{2 t^{2}}{\left(\tau \frac{L_{1}+L_{2}}{L_{1}}\right)^{2}}\right) \\
\quad \times\left[1+2 a \cdot \cos \left(2 \pi f_{m} t \cdot \frac{L_{1}}{L_{1}+L_{2}}\right)\right. \\
\left.\quad \cdot \cos \left(2 \pi^{2} L_{2} \beta_{2} \cdot \frac{L_{1}}{L_{1}+L_{2}} f_{m}^{2}\right)\right] .
\end{array}
$$




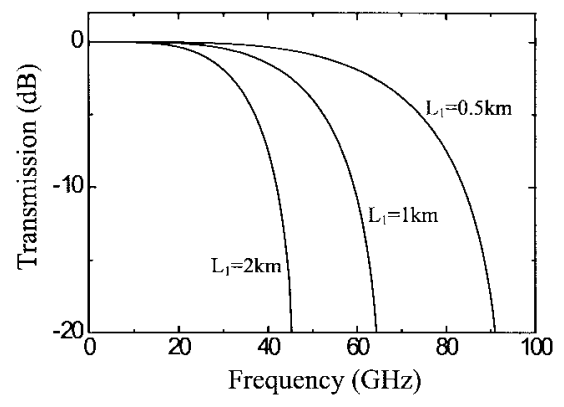

Fig. 3. Transmission characteristic of the time-stretch system for $M=10$ and for different values of the chirp rate (different $L_{1}$ ). The $x$ axis represents the input modulation frequency.

Equation (7) represents the transformation undertaken by the electrical signal during the time-stretch process. By comparing this intensity after dispersion with the intensity before the second dispersion, the following observations are made.

1) The pulse retains its Gaussian nature, and the Gaussian envelope is stretched by the factor given by

$$
M=1+L_{2} / L_{1} .
$$

2) The argument of the first cosine function shows that the modulation frequency has been reduced by the stretch factor $M$. The stretch factor is only dependent on the ratio of total dispersion in the first $\left(L_{1}\right)$ and second $\left(L_{2}\right)$ stages. In the experiment presented in Fig. 2, a length of $L_{1}=1.1 \mathrm{~km}$ has been used to generate the frequency chirp. According to (8), the stretch factor is 3, 6, and 7.9 for $L_{2}=2.2,5.5$, and $7.6 \mathrm{~km}$, respectively. Those values are in remarkable agreement with experimentally observed values of 3,6 , and 8 .

3) The intensity of the signal is reduced by the factor $M$. This results from the conservation of energy: a signal stretched in time by a factor $M$ is reduced in power by $M$.

4) The second cosine term represents the penalty introduced by the dispersion suffered in the second stage $L_{2}$.

The dispersion penalty occurs concurrent with the stretch process and limits the maximum frequency of the system. Although this phenomenon is qualitatively identical to the dispersion penalty experienced in a conventional analog link of lenth $L_{2}$, quantitatively, it is drastically different. According to (7), this process is described by the transfer function

$$
H_{\mathrm{att}}=\left(\cos \left(2 \pi^{2} \beta_{2} \frac{L_{2}}{M} f_{m}^{2}\right)\right)^{2} .
$$

The attenuation in intensity depends on the stretch factor $M$, the modulation frequency $f_{m}$, and the amount of chirp $1 / \beta_{2} L_{1}$. The attenuation appears due to the fact that the sidebands representing the modulation are phase shifted with respect to the carrier. Fig. 3 represents this low-pass characteristic for $M=10$ and for different lengths of fiber $L_{1}$. The $x$ axis represents the input RF frequency-the output frequency is actually divided by $M$. The higher the chirp rate (smaller $L_{1}$ ), the larger the bandwidth. For example, with a chirp obtained in $1 \mathrm{~km}$ of single-mode fiber (SMF) the

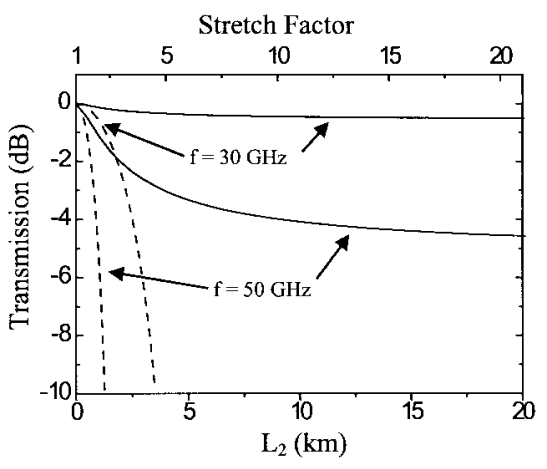

Fig. 4. Signal attenuation due to dispersion when propagating through the second fiber, for two different frequencies. Solid lines: optical carrier is chirped in $L_{1}=1 \mathrm{~km}$ of SMF. Dashed lines: single-frequency carrier.

bandwidth is more than $55 \mathrm{GHz}$, and it increases to more than $76 \mathrm{GHz}$ for $500 \mathrm{~m}$ SMF. However, reducing $L_{1}$ also reduces the aperture time of the system. The aperture time (i.e., segment of the input analog signal that can be captured and stretched in a single pass) is determined by the laser bandwidth multiplied by the total dispersion in $L_{1}$. With the 60-nm bandwidth for EDFL used in the experiments, the aperture time is approximately 1 and $0.5 \mathrm{~ns}$ for $L_{1}=1 \mathrm{~km}$ and 500 $\mathrm{m}$, respectively. Therefore, a tradeoff between the maximum analog bandwidth and the aperture time exists. Equation (9) also contains higher frequency peaks. To avoid the distortion caused by these peaks, the maximum signal bandwidth should be limited to the $-3 \mathrm{~dB}$ frequency described above.

While propagating through the second fiber, the envelope representing the signal is stretched and dispersed concurrently. Fig. 4 (solid lines) represents the attenuation due to dispersion as a function of $L_{2}$ (lower abscissa) and $M$ (upper abscissa) for modulation frequencies of 30 and $50 \mathrm{GHz}$, and for $L_{1}=1 \mathrm{~km}$. The dispersion penalty is small and quickly saturates as the signal propagates in the fiber. This phenomenon is expected, because, as the signal propagates, it is slowed down and becomes less sensitive to dispersion. The asymptotic value for large stretch factors depends on the modulation frequency. This behavior is in contrast to the classical dispersion occuring in the same length of fiber when the optical carrier is at a fixed wavelength (instead of being chirped). The conventional case corresponds to when $L_{1}$ tends to infinity in (8), and its effect can be expressed by the the transfer function $H_{\text {tran }}^{\prime}=$ $\left(\cos \left(\beta_{2} L_{2} \pi f_{m}^{2}\right)\right)^{2}$. The dashed lines in Fig. 4 represent the modulation amplitude at 30 and $50 \mathrm{GHz}$ when the signal is modulated on a fixed-wavelength carrier. Dispersion results in rapid and significant attenuation of the signal. By comparing the solid and dashed lines in Fig. 4, we observe that the dispersion penalty for the time-stretched signal is negligible compared to that of a conventional analog link with the same length of fiber

As a numerical example, we consider an EDFL with 60$\mathrm{nm}$ bandwidth. If we use $1 \mathrm{~km}$ of fiber to generate the chirp, then the aperture time is more than $1 \mathrm{~ns}$. For a stretch factor $M=10, L_{2}=9 \mathrm{~km}$ of fiber must be used for the second dispersion. According to (9), the 6-dB bandwidth of the system is approximately $55 \mathrm{GHz}$. Therefore, the output will be a 10-ns signal with a maximum frequency of $5.5 \mathrm{GHz}$. 


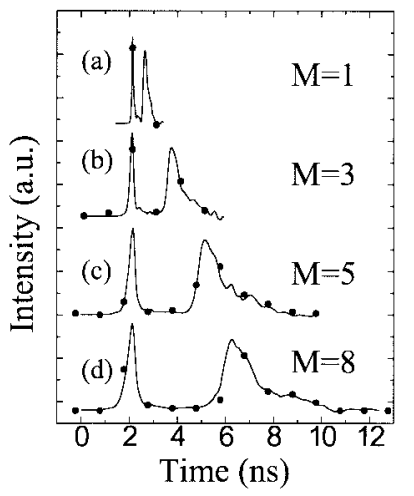

Fig. 5. Time-stretched ADC experiment consisting of the time-stretch preprocessor and a 1-Gsample/s electronic ADC.

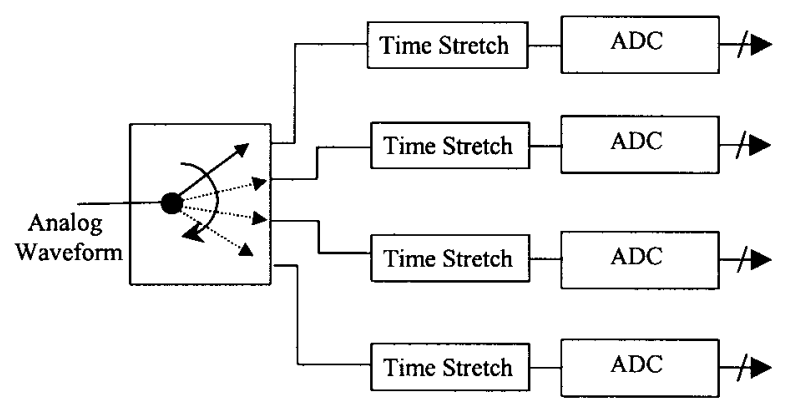

Fig. 6. System for stretching a continuous-time input signal. The input is segmented and interleaved into $M$ parallel channels, and each segment is stretched by $M$.

\section{ApPLICATION to ANALOG-TO-Digital (A/D) CONVERSION}

A time-stretch preprocessor can revolutionize A/D conversion. For a stretch factor $M$, the effective sampling rate and the input bandwidth of the ADC are increased by $M$ [1]. The preliminary A/D conversion experiment shown in Fig. 5 consists of the photonic time-stretch preprocessor and a 1-Gsample/s electronic ADC. The solid lines display the analog signal captured on the sampling oscilloscope (same as in Fig. 2), and the data points represent digitized samples produced by the electronic ADC. Slowing down the analog signal in time allows it to be captured by the ADC. For curve $(a)$ in Fig. 5, 1-ns spaced samples of the ADC are not sufficient to capture the waveform with $100 \mathrm{ps}$ transition time. In curves $(b)-(d)$ of Fig. 5, the effective sampling rate and the input bandwidth of the ADC are increased by 3,6 , and 8 , in proportion to the stretch factor $M$. In curve $(d)$ of Fig. 5, the 1Gsample/s ADC is nearly capable of capturing the high-speed analog signal with 100-ps transition times.

If the input analog signal is continuous in time, then a parallel architecture must be used in order to preserve the information. Fig. 6 shows the block diagram for such a system. The analog signal is segmented and interleaved into $M$ parallel channels. Each segment is time stretched by the same factor $M$ prior to entering a slow ADC. Segmentation in time creates sidebands in the signal spectrum. To ensure that the generated sidebands are below the quantization noise, the length of each segment, $T_{\mathrm{seg}}$, must be much greater than the period of the

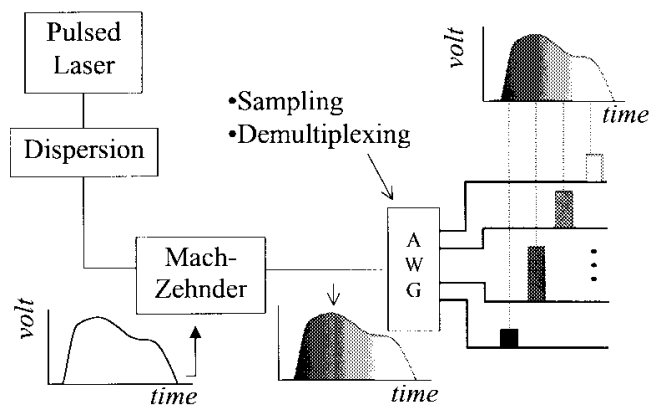

Fig. 7. WDS. A spatial-dispersive device such as an AWG replaces the second temporal-dispersive device (L2) in Fig. 1. The system performs sampling and interleaving in the wavelength domain and eliminates the need for high-speed switches.

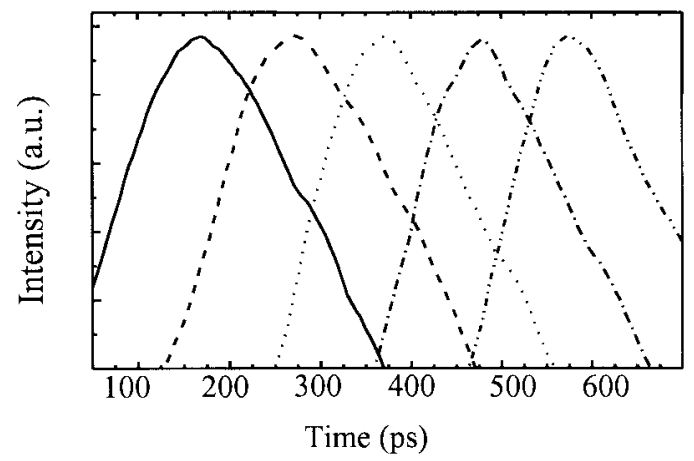

Fig. 8. Experimental data for WDS at 10 Gsamples/s.

carrier $T_{f}$. For example, $T_{\mathrm{seg}}>50 T_{f}$ is sufficient to ensure 10-bit accuracy.

\section{WDS}

An interesting system emerges if the second temporaldispersion stage in Fig. 1 is replaced by a spatial-dispersive element, such as a diffraction grating or an arrayed waveguide grating (AWG), as shown in Fig. 7 [10]. Each output port of the AWG filter samples a portion of the optical spectrum. Since each optical wavelength corresponds to a different time, the filter performs sampling in time. Pulses at the output of the filter correspond to a time-interleaved sampled version of the analog signal. This method of sampling eliminates the need for high-speed switches necessary in the traditional approach, where sampling is achieved via "time gating." Hence, problems caused by the limited speed and power dissipation of the switch are eliminated. Optical sampling techniques relying on the mixing of a narrow optical pulse with the fast electrical signal have been proposed and demonstrated [11]-[13]. Those techniques take advantage of the narrow pulsewidth available in optics to achieve ultrahigh timing resolution. However, they are based on the sampling oscilloscope technique and require a repetitive waveform. A real-time high-speed ADC system using the WDS would consist of the WDM sampler and a parallel array of electronic quantizers [10], [14]. Fig. 8 shows WDM sampling at 10 Gsamples/s. 160-fs full width at half maximum (FWHM) pulses were dispersed in $7 \mathrm{~km}$ of SMF producing a chirp of $120 \mathrm{ps} / \mathrm{nm}$. Sampling is performed by a silica AWG with a channel spacing of $0.8 \mathrm{~nm}$ and a 


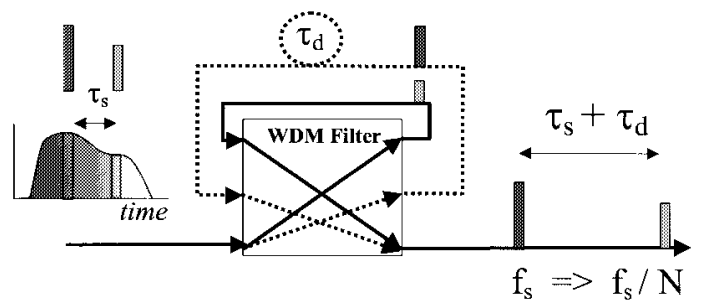

Fig. 9. The concept of TSWDS. The output is a sampled representation of the input, and the samples are slowed down in time by the AWG with feedback loop. The system represents the discrete-time version of the general time-stretching system shown in Fig. 1.

passband of $0.3 \mathrm{~nm}$. Outputs from individual AWG ports are detected and displayed using a digital-sampling oscilloscope. The measured pulse-to-pulse spacing of 100 ps agrees well with the $0.8 \mathrm{~nm}$ channel spacing of the AWG and the 120 $\mathrm{ps} / \mathrm{nm}$ chirp rate.

An interesting rendition of the WDS system is obtained if AWG outputs are fed back to the symmetric inputs with incremental delays, as shown in Fig. 9. Because of the input/output symmetry of the AWG, this feedback architecture represents a true time delay [15]. In the context of sampling, the feedback architecture separates the samples in time or, in effect, stretches the sampled waveform. The feedback converts a spatial-dispersive AWG to a temporal-dispersive device. The TSWDS system can, thus, be thought of as the discrete-time version of the general time-stretching system shown in Fig. 1. If the input analog signal is continuous in time, it must be segmented and fed into an array of TSWDS modules. The overall system architecture would be similar to Fig. 6.

\section{SUMMARY}

In summary, we have analyzed and demonstrated a timestretch system that can potentially enable A/D conversion of microwave and millimeter-wave signals. The system takes advantage of the tremendous bandwidth of ultrashort optical pulses to generate high-rate frequency chirp. We have derived and experimentally verified an expression for the stretch factor. It is shown that the dispersion penalty is reduced because the signal is slowed down as it propagates through the dispersive medium. In general, the dispersion penalty is negligible for high chirp rates. A discrete-time version of the system, performing sampling, can be realized if the dispersive fiber is replaced by an AWG with symmetric feedback.

\section{APPENDIX}

We derive the expression for the time-domain electric field after the second dispersion based on the assumption that $L_{1} \beta_{2} / \tau^{2} \ll 1$.

Let us consider one of the terms in (5)

$$
\begin{aligned}
T_{1}(f)= & \frac{\sqrt{\pi}}{\left|\sqrt{\tau^{-2}+j / 2 L_{1} \beta_{2}}\right|} \cdot \frac{a}{2} \\
& \cdot \exp \left[\left(\frac{-\pi^{2}\left(f-f_{m}\right)^{2}}{\tau^{-2}+j / 2 L_{1} \beta_{2}}\right)+j 2 \pi^{2} L_{2} \beta_{2} f^{2}\right] .
\end{aligned}
$$

We will try to simplify the exponent, $E_{1}$ of $T_{1}$. Since $L_{1} \beta_{2} / \tau^{2} \ll 1 E_{1}$ can be expressed in the following manner:

$E_{1} \approx j 2 \pi^{2} L_{1} \beta_{2}\left(f-f_{m}\right)^{2} \cdot\left(1+j 2 L_{1} \beta_{2} \tau^{-2}\right)+j 2 \pi^{2} L_{2} \beta_{2} f^{2}$.

Using $\gamma=1+j 2 L_{1} \beta_{2} \tau^{-2}$, (A2) can be rewritten as

$$
\begin{aligned}
E_{1}=j 2 \pi^{2} \beta_{2}\left(L_{1} \gamma+L_{2}\right) \\
\times\left[f^{2}-2 f \cdot f_{m} \frac{L_{1} \gamma}{L_{1} \gamma+L_{2}}+f_{m}^{2} \frac{L_{1} \gamma}{L_{1} \gamma+L_{2}}\right] .
\end{aligned}
$$

By noting that $2 L_{1} \beta_{2} \tau^{-2} \ll 1 \Rightarrow L_{1} \gamma /\left(L_{1} \gamma+L_{2}\right) \approx$ $L_{1} /\left(L_{1}+L_{2}\right)$, (A3) can be expressed as

$$
\begin{aligned}
E_{1}=j 2 \pi^{2} \beta_{2}\left(L_{1} \gamma+L_{2}\right) \times & \left(f-\frac{L_{1}}{L_{1}+L_{2}} \cdot f_{m}\right)^{2} \\
& +j 2 \pi^{2} L_{2} \beta_{2} \frac{L_{1}}{L_{1}+L_{2}} \cdot f_{m}^{2} .
\end{aligned}
$$

The term $T_{1}(f)$ can, therefore, be approximated by

$$
\begin{aligned}
T_{1}(f)= & \frac{\sqrt{\pi}}{\left|\sqrt{\tau^{-2}+j /\left(2 L_{1} \beta_{2}\right)}\right|} \\
& \cdot \frac{a}{2} \exp \left[\frac{-\pi^{2}\left(f-\frac{L_{1}}{L_{1}+L_{2}} f_{m}\right)^{2}}{\left.\left(\tau \frac{L_{1}+L_{2}}{L_{1}}\right)^{-2}+\frac{j}{2\left(L_{1}+L_{2}\right) \beta_{2}}\right]}\right. \\
& \times \exp \left(j 2 \pi^{2} L_{2} \beta_{2} \frac{L_{1}}{L_{1}+L_{2}} \cdot f_{m}^{2}\right) .
\end{aligned}
$$

From (A5), one can calculate the inverse Fourier transform of $T_{1}$

$$
\begin{aligned}
T_{1}(t)= & \frac{\mid \sqrt{\left(\tau \frac{L_{1}+L_{2}}{L_{1}}\right)^{-2}+\frac{j}{2\left(L_{1}+L_{2}\right) \beta_{2}} \mid}}{\left|\sqrt{\tau^{-2}+j /\left(2 L_{1} \beta_{2}\right)}\right|} \\
& \times \frac{a}{2} \exp \left[-t^{2} \cdot\left(\tau \frac{L_{1}+L_{2}}{L_{1}}\right)^{-2}\right] \\
& \times \exp \left(j 2 \pi \frac{L_{1}}{L_{1}+L_{2}} f_{m} t\right) \\
& \times \exp \left(j 2 \pi^{2} L_{2} \beta_{2} \frac{L_{1}}{L_{1}+L_{2}} \cdot f_{m}^{2}\right) \\
& \times \exp \left(\frac{-j t^{2}}{2\left(L_{1}+L_{2}\right) \beta_{2}}\right) .
\end{aligned}
$$

Similarly, we can treat other terms in (5) leading to (6).

\section{REFERENCES}

[1] A. S. Bhushan, F. Coppinger, and B. Jalali, "Time-stretched analogto-digital conversion," Electron. Lett., vol. 34, no. 9, pp. 839-840, 1998 .

[2] B. Jalali, F. Coppinger, and A. S. Bhushan, "Photonic time-stretch offers solution to ultrafast analog-to-digital conversion," Opt. Photon. News (Special Issue on Optics in 1998), pp. 31-32, Dec. 1998.

[3] F. Coppinger, A. S. Bhushan, and B. Jalali, "Time magnification of electrical signals using chirped optical pulses," Electron. Lett., vol. 34, no. 4, pp. 399-400, 1998

[4] W. J Caputi, "Stretch: A time-transformation technique," IEEE Trans. Aerosp. Electron. Syst., vol. AES-7, pp. 269-278, Mar. 1971. 
[5] B. H Kolner and M. Nazarathy, "Temporal imaging with a time lens," Opt. Lett., vol. 14, no. 12, pp. 630-632, June 1989.

[6] B. H. Kolner, "Generalization of the concepts of focal length and fnumber to space and time," J. Opt. Soc. Amer. A, Opt. Image Sci., vol. 11, no. 12, pp. 3229-3234, 1994.

[7] C. V. Bennet, E. P. Scott, and B. H. Kolner, "Temporal magnification and reversal of $100 \mathrm{~Gb} / \mathrm{s}$ optical data with an up conversion time microscope," Appl. Phys. Lett., vol. 65, no. 20, pp. 2513-2515, 1994.

[8] A. A. Godil, B. A. Auld, and D. M. Bloom, "Time-lens producing 1.9 ps pulses," Appl. Phys. Lett., vol. 62, no. 10, pp. 1047-1049, 1993.

[9] G. P. Agrawal, Nonlinear Fiber Optic. San Diego, CA: Academic, 1989.

[10] A. S. Bhushan, F. Coppinger, and B. Jalali, " 150 Gsample/s wavelength division sampler with time-stretched output," Electron. Lett., vol. 34, no 5, pp. 474-475, 1998.

[11] J. A. Valmanis and G. Mourou, "Electro-optic sampling: Testing picosecond electronics, Part 1. Principles and embodiments," Laser Focus, vol 22, no. 2, pp. 84-96, Feb. 1986.

[12] J. A. Valmanis and G. Mourou, "Electro-optic sampling: Testing picosecond electronics, Part 2. Applications," Laser Focus, vol. 22, no. 3, pp. 96-106, Mar. 1986.

[13] H. Takara, S. Kawanishi, T. Morioka, K. Mori, and M. Saruwatari, "100 Gbit/s optical waveform measurement with 0.6 ps resolution optical sampling using subpicosecond supercontinuum pulses," Electron. Lett., vol. 30, no. 14, pp. 1152-1154, 1994.
[14] M. Y. Frankel, J. U. Kang, and R. D. Esman, "High-performance photonic analogue-digital converter," Electron. Lett., vol. 33, no. 25 , pp. 2096-2097, 1997.

[15] S. Yegnanarayanan, P. D. Trinh, and B. Jalali, "Recirculating photonic filter: A wavelength-selective time delay for phased-array antennas and wavelength code-division multiple access," Opt. Lett., vol. 21, no. 10, pp. 740-742, 1996.

F. Coppinger, photograph and biography not available at the time of publication.

A. S. Bhushan, photograph and biography not available at the time of publication.

B. Jalali (S'86-M'89-SM'97), photograph and biography not available at the time of publication. 